\title{
Análise das complicações cardíacas associadas a COVID-19: uma revisão narrativa
}

\author{
Analysis of cardiac complications associated with COVID-19: a narrative review
}

Análisis de las complicaciones cardíacas asociadas con COVID-19: una revisión narrativa

Sandy Rodrigues de Carvalho ${ }^{1 *}$, Bruno Ferreira dos Santos ${ }^{1}$, Augusto César Campos da Silva ${ }^{1}$, Soly Guedes de Oliveira ${ }^{1}$, Meriam de Nazaré Marques Ferreira ${ }^{1}$, Janne de Jesus Bugarim Martins ${ }^{1}$, Tatiane Bahia do Vale Silva ${ }^{1}$, Nathalia Menezes Dias ${ }^{1}$, Shidney Salatiel Batista de Lima1, Wladyslaw Gryko Júnior².

\section{RESUMO}

Objetivo: Realizar uma revisão narrativa da literatura sobre as complicações cardíacas associadas a COVID19, assim como os efeitos que o vírus provoca no coração. Revisão bibliográfica: Dentre os achados na literatura a respeito das complicações cardíacas destacaram-se aspectos sobre os fatores de risco, relacionados não só a COVID-19 de forma geral, mas também ao sistema cardíaco, foco da pesquisa. Entre os fatores de risco a presença de DM, de HAS e idade avançada foram as mais frequentes. Além disso, as comorbidades mais encontradas foram as complicações por danos no miocárdio, alterações na Troponina I cardíaca, lesões cardíacas agudas, tromboses venosas e arritmias e estão, na maioria das vezes, relacionadas a mortes durante a hospitalização. Considerações finais: Percebeu-se a relevância das consequências da COVID-19 no sistema cardíaco em pacientes com ou sem DCV prévias. Compreender esses efeitos do vírus no sistema cardíaco possibilita a existência de uma melhor intervenção na atuação profissional, tendo como objetivo a redução de mortes causadas por comorbidades cardiovasculares.

Palavras-chave: COVID-19, Coronavírus, Doenças cardiovasculares.

\section{ABSTRACT}

Objective: This research aims to achieve a narrative review of the literature on cardiac complications associated with COVID-19, as well as the effects that the virus has on the heart. Bibliographic review: Among the findings in the literature regarding cardiac complications, aspects of risk factors were highlighted, related not only to COVID-19 in general, but also to the cardiac system, which is the focus of the research. Among the risk factors, the presence of DM, SAH and advanced age were the most frequent. In addition, the most common comorbidities were complications due to myocardial damage, changes in cardiac Troponin I, acute cardiac injuries, venous thrombosis and arrhythmias and are, in most cases, related to deaths during hospitalization. Final considerations: It concluded that the relevance of the consequences of COVID-19 on the cardiac system in patients with or without previous CVD was perceived. Understanding these effects of the virus on the cardiac system allows for a better intervention in professional practice, aiming to reduce deaths caused by cardiovascular comorbidities.

Keywords: COVID-19, Coronavirus, Cardiovascular diseases.

1 Universidade do Estado do Pará (UEPA), Tucuruí - PA. *E-mail: sandy.carvalho@aluno.uepa.br

2 Hospital Regional de Tucuruí (HRT), Tucuruí - PA.

SUBMETIDO EM: 9/2021

ACEITO EM: 10/2021

PUBLICADO EM: 10/2021 


\section{RESUMEN}

Objetivo: Realizar una revisión narrativa de la literatura en respecto a las complicaciones cardíacas asociadas con COVID-19, así como los efectos que tiene el virus en el corazón. Revisión bibliográfica: Entre los hallazgos de la literatura sobre las complicaciones cardíacas, se destacaron aspectos de los factores de riesgo, relacionados no solo con el COVID-19 en general, sino también con el sistema cardíaco, que es el foco de la investigación. Entre los factores de riesgo, la presencia de DM, HAS y la edad avanzada fueron los más frecuentes. Además, las comorbilidades más frecuentes fueron complicaciones por daño miocárdico, cambios en la troponina I cardíaca, lesiones cardíacas agudas, trombosis venosas y arritmias y están, en la mayoría de los casos, relacionadas con muertes durante la hospitalización. Consideraciones finales: Se constató la relevancia de las consecuencias del COVID-19 sobre el sistema cardíaco en pacientes con o sin ECV previa. Conocer estos efectos del virus sobre el sistema cardíaco permite una mejor intervención en la práctica profesional, con el objetivo de reducir las muertes por comorbilidades cardiovasculares.

Palabras clave: COVID-19, Coronavírus, Enfermedades cardiovasculares.

\section{INTRODUÇÃO}

Semelhante ao que ocorreu na China em 2002 com Síndrome Respiratória Aguda Grave (SARS) e em 2012 no Oriente Médio com a Síndrome Respiratória do Oriente Médio (MERS), a descoberta de um novo vírus em Wuhan, na China, no final de 2019, foi o responsável pelo surto de pneumonia denominado Síndrome Respiratória Aguda Grave do Coronavírus 2 (SARS-CoV-2)e pelo decreto de uma pandemia, em março de 2020, realizado pela Organização Mundial da Saúde (DANTAS KLS, et al., 2021; COSTA IBSS, et al., 2020).

A COVID-19 é a doença causada pelo vírus SARS-CoV-2 pertencente a família Coronavírus que vem infectando milhares de pessoas pelo mundo devido sua alta taxa de transmissão, realizada através de gotículas respiratórias que os indíviduos infectados liberam (FERRARI F, 2020; DANTAS KLS, et al., 2021). Os sintomas mais comuns são a fadiga, tosse seca, febre, falta de ar, congestão nasal e se o paciente possuir algum fator de risco, outros sistemas do organismo, além do respiratório, podem ser igualmente afetados (FERRARI F, 2020; DANTAS KLS, et al., 2021). Entre os fatores de risco, segundo um estudo realizado pelo Centro Chinês de Controle e Prevenção de Doenças, por Strabelli TMV e Uip DE (2020), se encontraram a Hipertensão Arterial Sistêmica (HAS), Doenças Cardiovasculares (DCV), Diabetes Mellitus e idade avançada.

Diversos estudos realizados analisaram os efeitos da COVID-19 no sistema cardíaco visto sua relevância quando se fala de mortalidade e tratamento. Dessa forma, observou-se que cerca de $1 / 4$ dos pacientes com COVID-19 apresentarão condições crônicas, sendo as DCV uma delas, o que acaba gerando um aumento cada vez maior referente ao número de óbitos por SARS-CoV-2 (STRABELLI TMV e UIP DE, 2020).

Segundo algumas pesquisas, essas complicações cardíacas podem ser resultado de diversos fatores, entre eles estão inclusos a inflamação sistêmica ou trombogênese, lesão cardíaca direta causada pelo vírus, isquemia relativa e alterações nos biomarcadores, estes relacionados com a maior probabilidade de o paciente chegar a óbito, como a Troponina I cardíaca e Dímero-D (COSTA IBSS, et al., 2020; OLIVEIRA GMM e PINTO FJ, 2020). Em contrapartida, outro artigo relatou não está claro ainda se as complicações cardíacas são provocadas principalmente pelo SARS-CoV-2 ou por essas condições multifatoriais devido a ocorrência de uma infecção sistêmica grave (MARTINS-FILHO PR, et al., 2020).

As comorbidades mais encontradas foram as complicações por danos no miocárdio, lesões cardíacas agudas, tromboses venosas e arritmias (STRABELLI TMV e UIP DE, 2020; OLIVEIRA GMM e PINTO FJ, 2020). Dessa forma, se torna evidente que indíviduos infectados pela COVID-19 e que se encontram em estágio grave da doença podem não só chegar a ter essas complicações cardiovasculares, como também podem ter choque de falência de diversos órgãos, o que acaba resultando no seu óbito (COSTA IBSS, et al., 2020).

Dessa forma, à vista do que foi mencionado, o presente artigo visou realizar uma revisão narrativa por meio da leitura de estudos mais relevantes sobre a temática que elucidem as principais complicações cardíacas associadas a COVID-19, bem como os efeitos que o vírus provoca no coração. 


\section{REVISÃO BIBLIOGRÁFICA}

\section{Fatores de Risco}

Dentre os achados na literatura a respeitos das complicações cardíacas destacaram-se aspectos sobre os fatores de risco, relacionados não só a COVID-19 de forma geral, mas também ao sistema cardíaco, foco da pesquisa (CHOW N, et al., 2020; COSTA IBSS, et al., 2020; MARTINS-FILHO PR, et al., 2020).

De acordo com resultados de um estudo preliminar realizado em março de 2020 nos Estados Unidos foi identificado que indivíduos que tinham a partir de 60 anos, portadores de DM, doença pulmonar crônica ou DCV, possuíam maior probabilidade de desenvolverem complicações e chegarem a óbito por COVID-19. Foram relatados 122.653 casos positivos para Coronavírus no estudo e desse total cerca de 7.162 apresentaram uma ou mais condições de saúde ou fatores de risco subjacentes. 10,9\% dessa porcentagem possuíam DM, 9,2\% doença pulmonar crônica e 9\% DCV. Ainda no mesmo artigo, relatou-se que dos 2.692 pacientes que possuíam uma ou mais comorbidades, 732 precisaram ser hospitalizados e 358 precisaram de Unidade de Terapia Intensiva (UTI) (CHOW N, et al., 2020).

A existência de DM tipo 2 em pacientes positivos para o vírus pode influenciar na gravidade dos sintomas que esses indivíduos apresentam e consequentemente, na sua recuperação (AL HAYEK A, et al., 2020). Após análise do prontuário eletrônico preenchido pela equipe médica em um estudo realizado na Arábia Saudita pelos autores Al Hayek A, et al. (2020), de 806 pacientes, 500 possuíam HAS, 195 apresentaram DCV e destes cerca de 387 precisaram de internação devido as complicações causadas pela COVID-19, principalmente naqueles pacientes com mais idade.

Em diversas pesquisas a relação idade avançada e comorbidades esteve presente (COSTA IBSS, et al., 2020; MARTINS-FILHO PR, et al., 2020; CHOW N, et al., 2020; AL HAYEK A, et al., 2020). Em um estudo realizado nos Estados Unidos, cerca de 425 indivíduos com idade igual ou maior que 65 anos e que apresentavam condições subjacentes relatadas precisaram ser hospitalizados, assim como outros 212 precisaram ser internados em uma UTI (CHOW N, et al., 2020).

Em consonância com isso, outro estudo realizado na Arábia Saudita relatou que pacientes com idade muito avançada apresentaram 12 vezes mais probabilidade de precisarem de uma hospitalização do que aqueles com idade abaixo dos 40 anos, relacionando isso ao fato de que devido as complicações já previamente existentes nessas pessoas, a infecção pela COVID-19 pode ser considerada um agravante para a situação de saúde dos mesmos (AL HAYEK A, et al., 2020). Isso é confirmado em outro artigo que relatou que pacientes idosos, pacientes que tinham DM ou HAS e pacientes com DCV prévias possuíram maior probabilidade de apresentarem danos no sistema cardíaco, assim como outro que cita como fatores de risco da COVID-19, além da idade avançada, a presença de HAS e DM (COSTA IBSS, et al., 2020; DANTAS KLS, et al., 2021).

Dessa forma, é possível inferir uma possível relação existente entre as complicações cardíacas e mortalidade, por esta ser um dos fatores de riscos da COVID-19. Em um estudo de coorte prospectivo realizado em dois hospitais de Wuhan, na China, de 191 pacientes estudados, mais da metade possuía alguma comorbidade, sendo HAS a mais comum (ZHOU F, et al., 2020). Zhou F, et al. (2020) ainda afirmaram que desses pacientes, 44 tinham insuficiência cardíaca e 28 vieram a óbito. Em outras pesquisas, de 500 pacientes que tinham HAS, quase $25 \%$ apresentaram DCV, da mesma forma que é relacionado que a taxa de letalidade de 44.726 casos positivos para SARS-CoV-2 foi de $10,5 \%$ para aqueles que tinham DCV e de $6 \%$ para aqueles que tinham HAS (CHOW N, et al., 2020; COSTA IBSS, et al., 2020).

Confirmando tais dados, um estudo observacional de séries temporais observou em suas análises que o número de óbitos associados a doenças hipertensivas em pacientes que tinham cerca de 20 a 59 anos, no ano de 2020 , aumentou em $21 \%$ se comparado com os quatro anos anteriores. Ademais, o mesmo estudo afirmou que entre os anos de 2019 e 2020, a taxa de letalidade intra-hospitalar referente a insuficiência cardíaca aumentou em 8\% e referente as doenças hipertensivas em 29\% (NORMANDO PG, et al., 2021). 
Nesse sentido, torna-se compreensível como complicações cardíacas podem está associadas com todos os fatores de risco existentes, mostrando como pacientes que possuem DCV podem ter resultados clínicos mais graves (BANSAL M, 2020). As complicações cardíacas mais encontradas nos estudos serão mais destrinchadas nos tópicos seguintes desta revisão.

\section{Danos no miocárdio e alterações na Troponina I Cardíaca}

Entre diversos estudos foi possível identificar problemas no miocárdio e lesão cardíaca aguda por alterações em biomarcadores como a Troponina I cardíaca. Strabelli TMV e Uip DE (2020) em sua pesquisa mostram que é possível observar que de 68 mortes confirmadas por COVID-19, 33\% foram resultados de dano no miocárdio e $7 \%$ de parada respiratória com dano miocárdico. De outros 138 pacientes positivos para COVID-19, 7,2\% apresentaram algum dano cardíaco com envolvimento de Troponina I de alta sensibilidade e alterações no ecocardiograma, o que revelou como está sendo comum nesses enfermos e como eles podem chegar a ter maiores riscos de mortalidade (ASKIN L, et al., 2020).

Os biomarcadores supracitados são importantes nas descobertas dessas complicações cardíacas, estando relacionados a diversos casos graves de infecção por COVID-19 (MACHAVA M, et al., 2020). Dentre esses biomarcadores se encontra uma família de proteínas chamada Troponinas, responsáveis pelo controle da contração muscular. Existem dois tipos de Troponinas que estão em pequenas quantidades no sangue e são encontradas apenas no coração, a Troponina I cardíaca e a Troponina T cardíaca. No entanto, quando alguma lesão no músculo cardíaco ocorre, esses níveis são alterados, se tornando mais elevados em pacientes graves da doença quando comparado a casos mais leves (ASKIN L, et al., 2020; DENG Q, et al., 2020; OLIVEIRA GMM e PINTO FJ., 2020).

Essas alterações de Troponina I cardíaca revelaram alta relação com a mortalidade de pacientes com COVID-19, tendo em um estudo atingido um pico dias antes de alguns pacientes chegarem a óbito e em outro está presente em pacientes com lesão cardíaca aguda (DENG Q, et al., 2020; BANSAL M, 2020). Em um indivíduo idoso sem DCV pré-existente que sentiu sintomas como miocardite fulminante ao ser analisado, também foi identificado níveis alterados de Troponina I cardíaca (ASKIN L, et al., 2020).

Da mesma forma, é afirmado no estudo de Martins JDN, et al. (2020), que independente do paciente apresentar histórico prévio de DCV ou não, o mesmo pode apresentar complicações relacionadas ao sistema cardíaco, sendo a miocardite uma das mais graves. Deng Q, et al. (2020), no entanto, refutaram ambas em sua pesquisa, relatando que apenas pacientes com DCV prévias ou com infarto agudo do miocárdio apresentaram exame ecocardiográfico com sinais típicos de miocardite durante a hospitalização.

Se entende como miocardite ou lesões cardíacas agudas os danos causados diretamente no músculo cardíaco, isso se dá como uma das respostas que o SARS-CoV-2 pode causar diretamente ou indiretamente no coração (ASKIN L, et al., 2020; OLIVEIRA GMM e PINTO FJ, 2020). Suspeita-se que a principal causa de morte dentro destes casos de COVID-19 sejam as miocardites e lesões cardíacas agudas em decorrência do SARS-CoV-2, onde esse acometimento se deve a replicação do vírus através da corrente sanguínea (DENG $Q$, et al., 2020). A existência de alterações eletrocardiográficas e elevação da Troponina em pacientes com COVID-19 que apresentavam miocardite deixou claro uma possível relação com a ocorrência de falência cardíaca aguda nos mesmos (COSTA IBSS, et al., 2020).

Outros estudos também mostraram que o desequilíbrio presente no sistema renina angiotensina secundária ao vírus e na Enzima Conversora de Angiotensina 2 (ECA2) é uma das principais causas de lesão cardiovascular, dessa forma, os danos no miocárdio estão diretamente ligados a ECA2 que está presente em altas quantidades no pulmão e coração (MARTINS JDN, et al., 2020).

É nessa enzima que a glicoproteína S do SARS-CoV-2 se liga ao receptor da ECA2 que ao se reproduzir, infecta as células e acaba alterando a enzima e diminuindo sua atividade, tornando mais vulneráveis pacientes com DCV já existentes e consequentemente, maior chance desses casos evoluírem de forma grave (COSTA IBSS, et al., 2020). Além disso, é mostrado que essas lesões não acontecem somente no coração, mas também em artérias e veias, tendo em um primeiro momento a probabilidade de gerar inflamações e em um segundo momento gerar trombos nas artérias do coração ou no cérebro (BANSAL M, 2020). 


\section{Tromboses}

O surgimento de trombos no organismo do paciente positivo para COVID-19 pode ocorrer devido não só uma inflamação no endotélio vascular, o que acaba prejudicando a regulação desse endotélio, mas também ao aumento da atividade pró-coagulante associado à pouca oferta de oxigênio nesse indivíduo (WANG C, et al., 2020; COSTA IBSS, et al., 2020). A hipercoagulação esteve presente em enfermos infectados pelo SARSCoV-2 e foi uma das maneiras de ocorrência das tromboses (HU T, et al., 2020). O processo de obstrução desses vasos ocorreu, na maioria das vezes, por coágulos sanguíneos, principalmente nos membros inferiores, cerca de $80 \%$ dos casos graves de pacientes com COVID-19 apresentaram trombose no pulmão. No entanto, não foi confirmado se essa coagulação estava se dando diretamente pelo SARS-CoV-2 ou por uma resposta imunológica exagerada na tentativa de cicatrizar as lesões causadas por ele (BANSAL M, 2020).

Por conseguinte, observou-se que o SARS-CoV-2 pode gerar tromboses não somente no coração, mas em todo o sistema cardiovascular. A maioria dos acometimentos de casos graves da doença tiveram algum tipo de trombo, seja ele arterial ou venoso, e a atenção para essas características se tornou indispensável no tratamento dos pacientes, haja vista que pacientes com COVID-19 estão mais propícios a esses eventos (BANSAL M, 2020). Ademais, a baixa taxa de mobilidade e as alterações na coagulação desses pacientes mostraram ser um fator de risco para a ocorrência de tromboses, tanto que em uma pesquisa realizada por Rossi FH (2020), acerca de tromboembolismo, é relatado sobre a importância de pacientes com suspeita de COVID-19 estarem sempre hidratados, terem uma boa alimentação e praticarem exercícios físicos (COSTA IBSS, et al., 2020; MACHAVA M, et al., 2020).

A trombose é comum entre as comorbidades causadas pela COVID-19 e pode ocorrer como trombose venosa aguda, trombose venosa das extremidades e embolia pulmonar crônica, podendo apresentar dor e edemas de membros inferiores, juntamente com dor torácica como manifestações clínicas (WANG C, et al., 2020; ROSSI FH, 2020).

A taxa de mortalidade em pacientes com eventos tromboembólicos foi $13,8 \%$ maior do que aqueles sem qualquer sinal de tromboses, da mesma forma, a taxa de tratamento na UTI para pacientes com trombose foi $15,9 \%$ maior do que aqueles que não tinham a complicação (WANG C, et al., 2020). Em pacientes hospitalizados ou em uma UTI a ocorrência de trombose é maior, haja vista que o SARS-CoV-2 está relacionado a um exagerado processo inflamatório (ROSSI FH, 2020). É também um dos motivos mais agravantes de morte juntamente com inflamação fulminante, isso revelou como a existência de tromboses esteve relacionada com um pior prognóstico e alta mortalidade da doença (ASKIN L, et al., 2020; ROSSI FH, 2020).

Apesar da idade avançada está mais relacionada com a ocorrência de diversas comorbidades, alguns pacientes mais jovens desenvolveram trombos arteriais e derrame de grandes vasos após a infecção por COVID-19 (HU T, et al., 2020). Ademais, há também a relação da obesidade como fator de risco para o surgimento de trombos em indivíduos com COVID-19 (DANTAS KLS, et al., 2021). O risco maior nesses casos, além de todos os outros comprometimentos do SARS-CoV-2, é que o coágulo continue na corrente sanguínea e acabe parando em algum órgão, como o coração, de forma fatal (MACHAVA M, et al., 2020).

\section{Arritmia}

Foi observado que pacientes com COVID-19 dentro dos fatores de risco podem apresentar arritmias como uma das complicações cardiovasculares, podendo surgir devido mudanças fisiológicas por alguma doença crítica, interação entre os remédios utilizados durante o tratamento do SARS-CoV-2 ou por comorbidades previamente existentes (KARAMCHANDANI K, et al., 2020). Da mesma forma, aqueles que além de apresentarem DCV subjacentes também possuírem histórico anterior de arritmias são mais suscetíveis a essa complicação (WANG Y, et al., 2020).

Ao se instalar na célula hospedeira e se reproduzir, o SARS-CoV-2 pode afetar o potencial de ação cardíaco e promover uma arritmia (WANG Y, et al., 2020). Alterações no intervalo QT também estão 
relacionados ao surgimento de arritmias, isso pode acontecer devido miocardite, distúrbios ou medicamentos, como a hidroxicloroquina, que por ter benefícios ainda incertos, esteve presente no prolongamento desse intervalo QT em pacientes com COVID-19, sendo necessário uma atenção redobrada para os remédios que serão aplicados e as possíveis alterações fisiopatológicas que podem provocar (WANG Y, et al., 2020; KARAMCHANDANI K, et al., 2020). Outros fatores de risco para surgimento da arritmia foram as associações realizadas a partir do surgimento de infecções virais como, por exemplo, a inflamação miocárdica, a ativação do sistema nervoso simpático e a disfunção metabólica (MACHAVA M, et al., 2020).

De acordo com os estudos, as isquemias miocárdicas, inflamação sistémica, distúrbios metabólicos, lesão cardíaca aguda e elevação nos níveis de troponina estavam associados significativamente com arritmias (HU T, et al., 2020; FERRARI F, 2020; COSTA IBSS, et al., 2020). Pacientes com níveis aumentados de Troponina tiveram maior incidência de arritmias ventriculares, maior necessidade de ventilação mecânica e foi relacionada também com evidências de mortalidade e gravidade, na qual sua elevação precedeu arritmias cardíacas e infartos ou miocardite fulminante (COSTA IBSS, et al., 2020).

Outros fatores como a gravidade da COVID-19, o tratamento na UTI para sustentar a vida dos pacientes e o diagnóstico realizado também podem desencadear o surgimento de arritmias (KARAMCHANDANI K, et al., 2020). Idosos com comorbidade também foram mais propensos a desenvolverem arritmias supraventriculares e fibrose atrial, isso se deu devido o envelhecimento do coração que ao formar um substrato favoreceu o surgimento de uma fibrilação atrial (KARAMCHANDANI K, et al., 2020). Além desses fatores, no estudo de Zhou F, et al. (2020) é mostrado que a arritmia juntamente com a insuficiência cardíaca ou infarto do miocárdio foram comuns em pacientes que estiveram em estado grave e possuíam pneumonia.

A arritmia pode ocorrer nesses pacientes como arritmias ventriculares ou braquias, taquicardia sinusal e bradicardia sinusal (WANG Y, et al., 2020). Em um estudo, entre os tipos de arritmias, as atriais foram as mais encontradas na UTI, a presença de inflamação sistêmica e possíveis causas subjacentes, como miocardite, podem estimular essa ocorrência (KARAMCHANDANI K, et al., 2020). Nesse sentido, a miocardite tem relação significativa com a presença de bradicardia e taquicardia (KARAMCHANDANI K, et al., 2020). Enfermos com COVID-19 possuem maior risco de terem arritmias malignas ou taquicardias ventriculares, podendo morrer não só por essas arritmias, como também por síndrome coronariana aguda e parada cardíaca (WANG Y, et al., 2020).

Pacientes com DCV prévias tem mais chances de terem arritmias ventriculares (KARAMCHANDANI K, et al., 2020). No entanto, na pesquisa de Hu T, et al. (2020) isso é refutado ao relatarem que as complicações cardíacas associadas com a infecção por SARS-CoV-2, incluindo elevação de Troponina, redução da função sistólica, miocardite e arritmia foram relatadas até mesmo em pacientes sem DCV preexistente.

Nesse sentido, identifica-se a relação mortalidade e a presença de arritmias nos estudos realizados. Alguns dados indicam que arritmia juntamente com lesão cardíaca aguda estavam presentes em quase $7 \%$ e $17 \%$ dos pacientes, respectivamente, sendo mais forte naqueles internados em uma UTI (FERRARI F, 2020). Em outra UTI, de todos os pacientes com COVID-19, 45\% tinham as arritmias como principal complicação (KARAMCHANDANI K, et al., 2020). Strabelli TMV e Uip DE (2020) citaram também em sua pesquisa que em uma avaliação de 138 pacientes internados, quase 17\% desenvolveram arritmia. A partir das pesquisas, entendeu-se que a arritmia necessita de atenção especial por ter relação com diversas complicações cardiovasculares (WANG C, et al., 2020).

\section{CONSIDERAÇÕES FINAIS}

De acordo com os achados percebe-se a relevância das consequências da COVID-19 no sistema cardíaco em pacientes com ou sem DCV prévias, tornando de suma importância que estudos continuem a analisar e demonstras casos clínicos para que as informações sejam cada vez mais debatidas e relatadas no meio científico. Compreender esses efeitos do vírus no sistema cardíaco possibilita a existência de uma melhor intervenção na atuação profissional e norteia as escolhas e estratégias traçadas no enfrentamento da atual pandemia, tendo como objetivo a redução de mortes causadas por essas comorbidades cardiovasculares. 


\section{REFERÊNCIAS}

1. AL HAYEK A, et al. Risk factors for hospital admission among COVID-19 patients with diabetes. A study from Saudi Arabia. Saudi Medical Journal, 2020; 41(10): 1090-1097.

2. ASKIN L, et al. O efeito da Doença de Coronavírus 2019 nas Doenças Cardiovasculares. Arquivos Brasileiros de Cardiologia, 2020: 114(5): 817-822.

3. BANSAL M. Cardiovascular disease and COVID-19. Diabetes \& Metabolic Syndrome: Clinical Research \& Reviews, 2020; 14(3): 247-250.

4. CHOW N, et al. Preliminary Estimates of the Prevalence of Selected Underlying Health Conditions Among Patients with Coronavirus Disease 2019 - United States, February 12-March 28, 2020. MMWR. Morbidity and Mortatlity Weekly Report, 2020; 69(13): 382-286.

5. COSTA IBSS, et al. O Coração e a COVID-19: O que o Cardiologista Precisa Saber. Arquivos Brasileiros de Cardiologia, 2020: 114(5): 805-816.

6. DANTAS KLS, et al. SARS-CoV-2: a view on its origin, transmission, genetic characteristics and pathogenicity. Revista Eletrônica Acervo Saúde, 2021; 13(6): 7567.

7. DENG Q, et al. Suspected myocardial injury in patientes with COVID-19: Evidence from front-line clinical observation in Wuhan, China. International Journal of Cardiology, 2020; 311: 116-121.

8. FERRARI F. COVID-19: Dados Atualizados e sua Relação com o Sistema Cardiovascular. Arquivos Brasileiros de Cardiologia, 2020; 114(5): 823-836.

9. HU T, et al. Cardiovascular Considerations in Coronavirus Disease 2019 with a Special Focus on Arrhythmia. Journal of Innovations in Cardiac Rhythm Management, 2020; 11(8): 4191-4198.

10. KARAMCHANDANI K, et al. Cardiac Arrhythmias in Critically III Patients With COVID-19: A Brief Review. Journal of Cardiothoracic and Vascular Anesthesia, 2020; 1-8.

11. MACHAVA M, et al. COVID-19 e Doença Cardiovascular: Uma Revisão da Literatura. Revista Moçambicana de Ciências de Saúde, 2020; 6(1): 64-67.

12. MARTINS-FILHO PR, et al. Biomarcadores de Lesão Miocárdica e Complicações Cardíacas Associadas à Mortalidade em Pacientes com COVID-19. Arquivos Brasileiros de Cardiologia, 2020; 115(2): 273-277.

13. MARTINS JDN, et al. As implicações da COVID-19 no sistema cardiovascular: prognóstico e intercorrências. J. Health Biol. Sci., 2020; 8(1): 1-9.

14. NORMANDO PG, et al. Redução na Hospitalização e Aumento na Mortalidade por Doenças Cardiovasculares durante a Pandemia da COVID-19 no Brasil. Arquivos Brasileiros de Cardiologia, 2021; 116(3): 371-380.

15. OLIVEIRA GMM, PINTO FJ. COVID-19: A Matter Close to the Heart. International Journal of Cardiovascular Sciences, 2020; 33(3): 1-4.

16. ROSSI FH. Tromboembolismo venoso em pacientes com COVID-19. Jornal Vascular Brasileiro, 2020; 19 : 1-5.

17. STRABELLI TMV, UIP DE. COVID-19 e o Coração. Arquivos Brasileiros de Cardiologia, 2020; 114(4): 598-600.

18. WANG C, et al. Prognosis of COVID-19 in patients with vein thrombosis: a systematic review and meta-analysis. European Review for Medical and Pharmacological Sciences,2020; 24: 10279-10285.

19. WANG Y, et al. Cardiac arrhythmias in patients with COVID-19. Journal of Arrhythmia, 2020; 36(5): 827-836.

20. ZHOU F, et al. Clinical course and risk factors for mortality of adult in patients with COVID-19 in Wuhan, China: a retrospective cohort study. The Lancet, 2020; 395(10229): 1054-1062. 